\title{
Fit for Purpose - Standardisierung von inhaltserschließenden Informationen durch Richtlinien für Metadaten
}

\section{Einleitung}

Schwieriger war dann doch zu entscheiden, welche der optionalen Metadatenfelder ich ausfüllen will. Eigentlich ist mir klar, dass die möglichst detaillierte Beschreibung das Finden und die Nachnutzung der Daten erleichtert. Trotzdem war ich bei einigen Feldern ratlos und ließ sie leer. ${ }^{1}$

In diesem Auszug eines Berichts einer Forscherin aus dem Feld der Digital Humanities über die erste Veröffentlichung von Forschungsdaten auf einem Repositorium aus dem Jahr 2017 wird der herausragende Stellenwert von beschreibenden Informationen von einer Ressource in strukturierter Form deutlich betont. Durch adäquate Angaben soll ein Auffinden und somit die potenzielle Nachnutzung von digitalen Forschungsdaten durch andere Nutzende ermöglicht werden. Neben dem Mehrwert einer umfassenden Auszeichnung mittels Metadaten wird gleichzeitig eine hierfür erforderliche Kompetenz zuerkannt. In diesem konkreten Fall, um irrige Angaben zu vermeiden und somit die Wertigkeit der bereits bereitgestellten Metadaten nicht zu relativieren, mündete dies in einem Verzicht auf weitere, eigene Informationsangaben.

Die hier geschilderte Erfahrung steht im Einklang mit den Lessons learned Thesen zur FDM-Kompetenzausbildung aus dem Jahr 2019, wonach „Erfahrungen mit der Beschreibung mit Metadaten oder gar der Veröffentlichung von Forschungsdaten [...] bei ihnen [den kontaktierten Graduierten] in der Regel noch gar nicht vor[lagen].“2 Zwar ist eine „Verständigung darüber, welchen Begriff

1 Mache, Beata: Meine erste Kollektion im DARIAH-DE Repository. DHdBlog. 7.12.2017. https:// dhd-blog.org/?p=8798 (16.10.2020).

2 Einwächter, Sophie G. u. a.: Lessons learned - Thesen zur FDM-Kompetenzausbildung: Erkenntnisse aus dem interdisziplinären Vernetzungstreffen der vom BMBF geförderten Projekte eeFDM Jena, FDMentor, FOKUS, PODMAN und UniLLAB am 30. und 31. Januar 2019 in Marburg. In: Bausteine Forschungsdatenmanagement (2020) 1. S. 8-15, https://doi.org/10.17192/ bfdm.2020.1.8101, S. 12. Ferner s. DFG: Leitlinien zur Sicherung guter wissenschaftlicher Praxis - Kodex. Bonn 2019. https://www.dfg.de/download/pdf/foerderung/rechtliche_rahmenbe dingungen/gute_wissenschaftliche_praxis/kodex_gwp.pdf (16.10.2020), S. 17-19. Zu Kompetenzen s. Allianz der deutschen Wissenschaftsorganisationen: „Wege zur digitalen Qualifikation“: ein Diskussionspapier. 2020. https://doi.org/10.2312/allianzoa.038. S. exemplarisch Ba-

๖ Open Access. (C) 2021 Joachim Laczny, publiziert von De Gruyter. (๕) Br-Nc-ND Dieses Werk ist lizenziert unter der Creative Commons Attribution-NonCommercial-NoDerivatives 4.0 Lizenz. 
von Forschungsdaten beispielsweise die Geisteswissenschaften haben, [...] so nach wie vor Gegenstand der Diskussion",3 obgleich seitens der historisch arbeitenden Wissenschaften zuerkannt wird, dass die „Qualität der von Archiven und Bibliotheken bereitgestellten Erschließungsdaten als Forschungsdaten [...] hier eine hohe Bedeutung [besitzt], denn bei der Erstellung von Metadaten beginnt in vielen Fällen bereits der Forschungsprozess.“4

Im Folgenden soll daher der Frage nachgegangen werden, inwiefern Bibliotheken den Qualitätsanspruch an inhaltserschließende Informationen von Ressourcen durch die Formulierung und Veröffentlichung einer bibliotheksspezifischen, übergeordneten Metadaten-Richtlinie bzw. -Policy - auch im Sinne einer Transparenzoffensive - und deren Anwendung beeinflussen können.

\section{Datenqualität}

Bis zum Jahr 2010 wurde das Themenfeld der Datenqualität oft als „anecdotal

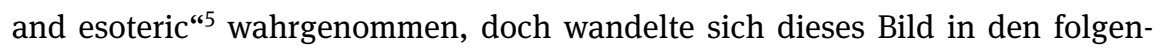
den Jahren vor dem Hintergrund von Big Data und verstärkter Forschung im Bereich der Data Quality. ${ }^{6}$ Einen Zugang bieten die von Wang und Strong 1996 vorgestellten Qualitätsdimensionen. Dieses Conceptual Framework of Data Quality umfasst vier Datenqualitätsdimensionen, die sich aufgliedern in intrinsic, contextual, representational und accessibility. Der erste Bereich beinhaltet die Aspekte believability, accuracy, objectivity und reputation; der zweite value-added, relevancy, timeliness, completeness und appropriate amount of data; der dritte

latsoukas, Panos u. a.: A Method for Examining Metadata Quality in Open Research Datasets Using the OAI-PMH and SQL Queries: the Case of the Dublin Core 'Subject' Element and Suggestions for User-Centred Metadata Annotation Design. In: International Journal of Metadata, Semantics and Ontologies (2018) 13,1. S. 1-8, https://doi.org/10.1504/IJMSO.2018.096444, S. 3, 6. Ferner Broeder, Daan u. a.: SSHOC D3.1 Report on SSHOC (meta)data interoperability problems. 10.12.2019. https://doi.org/10.5281/zenodo.3569868, S. 18-20; zu kontrollierten Vokabularen s. S. 27. Eher polemisch: Doctorow, Cory: Metacrap: Putting the torch to seven straw-men of the meta-utopia. 26.8.2001. https://people.well.com/user/doctorow/metacrap.htm (16.10.2020).

3 Lessons learned - Thesen zur FDM-Kompetenzausbildung, S. 11.

4 Paulmann, Johannes und Eva Schlotheuber: Digitale Wissensordnung und Datenqualität: Herausforderungen, Anforderungen und Beitrag historisch arbeitender Wissenschaften. In: Archivar (2020) 73,1. S. 9-12, S. 11.

5 Shankaranarayanan, G. und Roger Blake: From Content to Context: The Evolution and Growth of Data Quality Research. In: Journal of Data and Information Quality (2017) 8,2. S. 1-28, https://doi.org/10.1145/2996198, S. 2.

6 S. From Content to Context, S. 2. 
interpretability, ease of understanding, representational consistency und concise representation; der letzte accessibility und access security. ${ }^{7}$

Einige der mittlerweile sehr zahlreichen Forschungsfelder im Bereich der Data Quality im Zusammenhang mit den verschiedenen Dimensionen wurden 2017 analysiert, wobei insgesamt eine Forschungstendenz zu beobachten ist, die sich ausgehend von content-based stärker zu context-based hinwendet, wie die Einbeziehung von Qualität in Systementwicklungsprozesse zeigt. ${ }^{8}$ U. a. wurde die Bedeutung zwischen bereitgestellten Metadaten für Primärdaten hinsichtlich der believability und der fitness-for-use in einem Ansatz aus dem Jahr 2003 betont, um unter Berücksichtigung dieser beiden Aspekte den Zugang für Nutzende zu verbessern. ${ }^{9}$ Auch im Bibliothekswesen wird im Zusammenhang mit Analysen auf verschiedene Qualitätsdimensionen bzw. -aspekte zurückgegriffen. ${ }^{10}$

Im Jahr 2019 resümierte jedoch der Rat für Informationsinfrastrukturen in der Publikation Herausforderung Datenqualität - Empfehlungen zur Zukunftsfähigkeit von Forschung im digitalen Wandel, dass die Begrifflichkeit Datenqualität in der Wissenschaft nur schwierig zu bestimmen, wie auch über externe bzw. politische Vorgaben nur äußerst bedingt zu steuern sei. Auch eine Normierung von Datenqualität entsprechend etwaiger DIN- bzw. ISO-Vorgaben erscheine aufgrund der Komplexität digitaler Forschungsprozesse wenig zielführend. ${ }^{11}$

7 S. Wang, Richard Y. und Diane M. Strong: Beyond Accuracy: What Data Quality Means to Data Consumers. In: Journal of Management Information Systems (1996) 12,4. S. 5-33, https:// www.jstor.org/stable/40398176, S. 20-21. Ferner Batini, Carlo und Monica Scannapieca: Data quality: concepts, methodologies and techniques. Berlin, New York: Springer 2006 (Datacentric systems and applications), S. 19-49. Ochoa, Xavier und Erik Duval: Automatic Evaluation of Metadata Quality in Digital Repositories. In: International Journal on Digital Libraries (2009) 10. S. 67-91, https://doi.org/10.1007/s00799-009-0054-4, S. 69-70.

8 S. From Content to Context, S. 18-19, 25.

9 S. für einen Ansatz Shankar, G. und Stephanie Watts: A relevant, believable approach for data quality assessment. In: Proceedings of the Eighth International Conference on Information Quality. 2003. S. 178-189, http://mitiq.mit.edu/ICIQ/Documents/IQ\%20Conference\%202003/ Papers/ARelevantBelievableApproachForDQAssessment.pdf (16.10.2020).

10 Exemplarisch s. Gentry, Steven u. a.: Survey of Benchmarks in Metadata Quality: Initial Findings. 2020. https://digital.library.unt.edu/ark:/67531/metadc1637685/ (16.10.2020), S. 1921, 34. Für etwaige Evaluationsszenarien, auch unter Beteiligung von Nutzenden, s. Golub, Koraljka u. a.: A Framework for Evaluating Automatic Indexing or Classification in the Context of Retrieval. In: Journal of the Association for Information Science and Technology (2016) 67,1. S. 3-29, https://doi.org/10.1002/asi.23600.

11 S. Rat für Informationsinfrastrukturen (Hrsg.): Herausforderung Datenqualität. Empfehlungen zur Zukunftsfähigkeit von Forschung im digitalen Wandel. 2. Aufl. Göttingen 2019, http:// www.rfii.de/?p=4043 (16.10.2020), S. 28. Für die Diskussion von fünf Leitideen der Qualitätssteuerung von Forschungsdaten s. Herausforderung Datenqualität, S. 15. 
Als Mittelweg wird daher eine pragmatische Normierung unter dem Blickwinkel fit for purpose als nutzendenzentriertes Ziel aufgezeigt, um die Verwendbarkeit von Daten ins Zentrum zu rücken. ${ }^{12}$ Unter dem Titel Fitness for purpose: the future evolution of bibliographic records and their delivery lenkte Ede 1995 das Augenmerk aus Sicht der British Library auch auf diesen Aspekt hin, wobei Qualität als ein Synonym von ,accuracy and content“13 für bibliografische Aufnahmen verstanden wurde.

\section{Metadaten}

Nach einer weit gefassten Definition sind „Metadaten allgemein verstanden Aussagen über jede beliebige, identifizierbare, benennbare, adressierbare oder in irgendeiner Weise handhabbare Entität unserer Welt" ${ }^{* 14}$ und somit werden im Folgenden inhalts- bzw. sacherschließende Informationen über Ressourcen diesen ebenfalls zugeordnet.

Zwar wird es „[m]it der steigenden Bedeutung der Metadaten im Forschungsprozess [...] notwendig, dass diese ebenfalls Gegenstand von Qualitätssicherungsprozessen werden " ${ }^{15}$ dennoch sind Fragen nach Aufwand und Nutzen von Beschreibungssystemen wie auch die Auswahl aus den vielfältigen Beschreibungspfaden und -sprachen zu beantworten und letztendlich stellt sich „[i]nsgesamt [...] der Beschreibungsaufwand in der Praxis als entscheidende Hürde dar“. 16

12 Herausforderung Datenqualität, S. 25, 28. „The concept of 'fitness for use' is now widely adopted in the quality literature. It emphasizes the importance of taking a consumer viewpoint of quality because ultimately it is the consumer who will judge whether or not a product is fit for use." Beyond Accuracy, S. 6.

13 Ede, Stuart: Fitness for purpose: the future evolution of bibliographic records and their delivery. In: Catalogue and Index (1995) 116. S. 1-3, S. 1.

14 Gradmann, Stefan: Container - Content - Context: zur Evolution bibliothekarischer Metadaten von Katalogdaten zu Library Linked Data. In: Handbuch Bibliothek: Geschichte, Aufgaben, Perspektiven. Hrsg. von Konrad Umlauf und Stefan Gradmann. Stuttgart, Weimar: Metzler 2012. S. 121-128, S. 121. Exemplarisch s. Riley, Jenn: Understanding Metadata: What Is Metadata, and What Is It For? Baltimore 2017. http://www.niso.org/publications/understanding -metadata-riley (16.10.2020). Howarth, Lynne C.: Metadata and Bibliographic Control: SoulMates or Two Solitudes? In: Cataloging \& Classification Quarterly (2005). 40,3-4. S. 37-56, https://doi.org/10.1300/J104v40n03_03, S. 40-42.

15 Herausforderung Datenqualität, S. 37.

16 Herausforderung Datenqualität, S. 38. „As Jones et al. (2006) observe, the key is 'to find the balance of responsibility for documenting data between individual researchers and trained data stewards who have advanced expertise with appropriate metadata standards and techno- 
Die Verknüpfung von Daten aus unterschiedlichen Zusammenhängen und Disziplinen verspricht durchaus einen äußerst starken Anreiz für intra- und interdisziplinäre Forschungsprojekte. Doch geht dies aufgrund der häufig heterogenen Struktur und Semantik der vorhandenen Daten mit nicht minder großem Aufwand einher. Nicht förderlich in diesem Kontext sind zudem die häufig in den Fachdisziplinen fehlenden Definitionen zur Strukturierung von Daten bzw. Inhalten oder auch die ,verwirrende Vielzahl an anwendbaren Spezifikationen und Metadatenstandards, was es selbst Spezialisten erschwert, breit verfügbare Datenbestände aufzubauen“. ${ }^{17}$ Als Mehrwert bleibt zwar festzuhalten, dass in der Datenaufbereitung oft Verbesserungen stattfinden, doch „Praxisprobleme betreffen vor allem Fragen von Datenstruktur und -integrität. [...] Die Diskrepanz zwischen den theoretischen technischen Möglichkeiten und dem in der Praxis notwendigen Aufwand einer solchen Integration ist allerdings noch erheblich."18

Das bibliografische Umfeld bleibt hiervon nicht unberührt und die Ausprägungen von Standards und Datenmodellen bzw. Richtlinien sind ebenfalls vielfältig und in stetem Wandel begriffen. ${ }^{19}$ Pointiert lassen sich die derzeitigen Entwicklungen $\mathrm{zu}$ bibliografischen Datenmodellen nach Suominen jedoch wie folgt zusammenfassen: „If interoperability of bibliographic Linked Data is a goal, then the defining of new data models needs to stop. “20

Gleichzeitig streben Bibliotheken im Zuge der digitalen Transformationsprozesse danach, die Datenqualität im Bereich der Metadatenerstellung und

logies.'“ Peer, Limor u. a.: Committing to Data Quality Review. In: International Journal of Digital Curation (2014) 9,1. S. 263-291, https://doi.org/10.2218/ijdc.v9i1.317, S. 281. Perspektivisch zu Forschungssoftware und Instrumenten s. Bach, F. u. a.: Muster-Richtlinie Nachhaltige Forschungssoftware an den Helmholtz-Zentren. Potsdam 2019. https://doi.org/10.2312/OS.HELM HOLTZ.007. Stocker, Markus u.a.: Persistent Identification Of Instruments. In: arXiv:2003.12958 [cs-DL], 29.3.2020. http://arxiv.org/abs/2003.12958.

17 Herausforderung Datenqualität, S. 46. Für einen grafischen Überblick s. Riley, Jenn: Seeing Standards: A Visualization of the Metadata Universe. 2010. http://jennriley.com/metadatamap/ (16.10.2020). S. exemplarisch Schulz, Julian u. a.: Standardisierung eines Standards: Warum und wie ein Best-Practice-Guide für das Metadatenschema DataCite entstand. In: Korpus im Text (2020). http://www.kit.gwi.uni-muenchen.de/? $p=42800 \& v=1$ (16.10.2020).

18 Herausforderung Datenqualität, S. 45, 46.

19 S. exemplarisch: Figure 1: Family forest of bibliographic data models. In: Suominen, Osma u. a.: From MARC Silos to Linked Data Silos? In: o-bib. Das offene Bibliotheksjournal (2017) 4,2. S. 1-13, https://doi.org/10.5282/O-BIB/2017H2S1-13, S. 2; Riva, Pat u. a.: IFLA Library Reference Model (LRM). Den Haag 2017. https://www.ifla.org/files/assets/cataloguing/frbr-lrm/ifla-lr m-august-2017_rev201712.pdf (16.10.2020).

20 From MARC Silos to Linked Data Silos?, S. 11. 
-pflege zu konsolidieren bzw. zu steigern. ${ }^{21}$ Dennoch bleibt die Aussage zutreffend: „[...] what quality cataloging is lies partially in the subjective dimensions of the definition. While accuracy is seldom disputable, the needs of users are varied, and there is insufficient documentation of what these needs are."22 Auch Fragen zur Erschließung und Vernetzung der Metadaten im Zusammenspiel mit den Digital Humanities rücken in den Vordergrund. ${ }^{23}$ Doch rechtliche Vorgaben umrahmen stets die Handlungsspielräume für die Metadatenbearbeitung und Nachnutzung. ${ }^{24}$

Im Zusammenhang mit einer Schärfung der Begrifflichkeit Datenqualität im bibliothekarischen Umfeld liegt ein Streben nach der Erstellung bzw. Pflege eines perfekten Katalogisats äußerst nahe und wurde folglich mehrfach thematisiert. ${ }^{25}$ Nicht selten findet laut Bade das perfekte Katalogisat Erwähnung, allerdings oft vor dem Hintergrund, eine Diskussion um Qualitätskriterien zu vermeiden, denn „In these and many other texts 'the perfect record' is simply a rhetorical strategy for dismissing all issues concerning quality by reducing the very complex and context dependent notion of quality to what is implied in the phrase 'the perfect record'. It is a phrase used almost entirely by those who categorically reject it in the context of demands for or questions concerning quality. “26

Letztendlich sei dabei eine Diskussion um unvollkommene oder vermeintlich makellose Aufnahmen nicht zielführend, sondern vielmehr zunächst die auf institutioneller Ebene ehrliche Beantwortung folgender zwei Fragen:

1. What data elements are useful for the kind of library research performed here in this particular institution?

2. How much, and which elements of that necessary information can this institution afford to support? (This means either creating it initially, correcting or adding it to

21 S. Herausforderung Datenqualität, S. 71. Franke-Maier, Michael: Anforderungen an die Qualität der Inhaltserschließung im Spannungsfeld von intellektuell und automatisch erzeugten Metadaten. In: ABI-Technik (2018) 38,4. S. 327-331, https://doi.org/10.1515/abi tech-2018-4005, S. 327-328.

22 Thomas, Sarah E.: Quality in bibliographic control. In: Library Trends (1996) 44,3. S. 491505, S. 494.

23 S. Döhl, Frédéric: Digital Humanities und Bibliotheken. Über technisch-organisatorische Infrastruktur hinausgedacht. In: Zeitschrift für Bibliothekswesen und Bibliographie (2019) 66,1. S. 4-18, https://doi.org/10.3196/186429501966114, S. 9.

24 S. Klimpel, Paul: Kulturelles Erbe digital: eine kleine Rechtsfibel. Berlin: digiS Forschungsund Kompetenzzentrum Digitalisierung 2020. https://doi.org/10.12752/2.0.004.0, S. 27-31.

25 S. Bade, David: The Perfect Bibliographic Record: Platonic Ideal, Rhetorical Strategy or Nonsense? In: Cataloging \& Classification Quarterly (2008) 46,1. S. 109-133, https://doi.org/ 10.1080/01639370802183081, S. 113-118.

26 The Perfect Bibliographic Record, S. 114. 
bibliographic records imported from external sources, and future maintenance in cases of changing standards, new headings, data definitions, etc. $)^{27}$

Auch in anderen kulturgutverwahrenden Institutionen rückt, wie während der Erschließung von Archivbeständen - unter Anwendung einer Normdatei -, die Definition des Qualitätsbegriffs von Daten in die nähere Betrachtung, denn die „Bezeichnung Datenqualität bzw. die Aussage darüber, ob Daten eine geringere oder höhere Qualität haben, ist zunächst sehr frei und liegt im Auge des Betrachters; denn sie ist abhängig vom Anwendungsfall, in dem die Nutzung von Daten vorgestellt wird. Den Anwendungsfall als Maßstab für Datenqualität zu nehmen, hat zwei charmante Seiten: eine empirische und eine ökonomische. “28

\section{Prinzipen und Richtlinien}

Neben den FAIR Data Principles für nachhaltig nachnutzbare Forschungsdaten, welche findable, accessible, interoperable, and re-usable sein sollen, ${ }^{29}$ wurden 2020 speziell für Metadaten ebenfalls vier Prinzipien durch die Initiative Metadata 2020 vorgestellt: compatible, complete, credible, curated. ${ }^{30}$

Im Detail bedeutet dies für das erste Prinzip compatible, dass ein Leitfaden für die Lesbarkeit von Maschine und Mensch bereitgestellt wird. Metadaten müssen daher so offen, interoperabel, durch Maschinen bearbeitbar sowie maschinen- und von Menschen lesbar wie möglich sein. Das zweite Prinzip complete fordert die Darlegung des Inhalts, der Bestandteile und Beziehungen, wie diese publiziert wurden. Folglich müssen Metadaten möglichst umfassend und vollständig sein. Das dritte Prinzip credible verlangt, die Auffindbarkeit und Langlebigkeit der Inhalte zu gewährleisten. Somit müssen die Metadaten von

27 The Perfect Bibliographic Record, S. 129. S. exemplarisch Report and Recommendations from the Europeana Task Force on Metadata Quality. Den Haag 2015. http://pro.europeana. eu/files/Europeana_Professional/Publications/Metadata\%20Quality\%20Report.pdf (16.10.2020), S. 43.

28 Müller, Gerhard: Die Normdatei als ein Mittel in der Erschließung von Archivbeständen. In: Brandenburgische Archive (2015) 32. S. 8-16, S. 11.

29 S. Wilkinson, Mark D. u. a.: The FAIR Guiding Principles for Scientific Data Management and Stewardship. In: Scientific Data (2016) 3. 160018, https://doi.org/10.1038/sdata.2016.18. S. zuletzt für Repositorien Lin, Dawei u. a.: The TRUST Principles for Digital Repositories. In: Scientific Data (2020) 7,144. https://doi.org/10.1038/s41597-020-0486-7.

30 S. Kaiser, Kathryn u. a.: Methods \& Proposal for Metadata Guiding Principles for Scholarly Communications. In: Research Ideas and Outcomes (2020) 6. e53916. https://doi.org/10.3897/ rio.6.e53916, S. 5. Für sieben „Key Elements of Good Quality Metadata“ s. Report and Recommendations from the Europeana Task Force on Metadata Quality, S. 8-31. 
eindeutiger Herkunft, vertrauenswürdig und von Exaktheit beschaffen sein. Das letzte Prinzip curated trägt einer notwendigen Aktualisierung und Veränderung Rechnung und folglich sind Metadaten im Laufe der Zeit angemessen zu ergänzen, zu pflegen bzw. zu migrieren.

Ausgehend von diesen Prinzipien werden acht Handlungsempfehlungen abgeleitet, wonach $\mathrm{u}$.a.

- vorhandene Metadatenschemata und deren Anwendung favorisiert

- Best-Practice-Elemente genutzt, Metadaten als strategischer, primärer Output - folglich wie Inhalte - aufgefasst

- regelmäßige Datenprüfungen und Modifikationen zur Verringerung von fehlenden Angaben und Fehlern durchgeführt

- Persistente Identifikatoren (PID) und eine normalisierte Inhaltsbeschreibung im Sinne des Best Practice angewendet

- Anfragen von Nutzenden zur Datenkorrektur beantwortet

- Feedback-Mechanismen für Nutzende etabliert und

- eine kollaborative Arbeitsweise angestrebt werden sollen. ${ }^{31}$

Um Metadaten bzw. Erschließungsinformationen einer Bibliothek entsprechend der vorangegangenen Prinzipien für eine intra- und interdisziplinäre (Nach-) Nutzung, nicht nur in Katalog- bzw. Discovery-Systemen, interoperabel und nutzendenzentriert bereitzustellen, können entsprechende Anforderungen im Rahmen von Strategieprozessen einer Institution durch die Formulierung und Veröffentlichung von Metadaten-Richtlinien bzw. -Policies somit vertieft werden.

So definiert die Niedersächsische Staats- und Universitätsbibliothek (SUB) Göttingen wie folgt:

1. Die Qualität von Informationsdienstleistungen und der dazu eingesetzten Informationssysteme bemisst sich nach der Fähigkeit, zutreffende und verlässliche Antworten geben zu können.

2. Diese Fähigkeit hängt direkt von der Qualität der dafür genutzten Metadaten ab.

3. Die von der SUB Göttingen erzeugten, bearbeiteten sowie genutzten Metadaten müssen daher hohen Qualitätsanforderungen entsprechen, um die Qualität

a) eigener Informationsdienstleistungen sowie

b) Informationsdienstleistungen Dritter, die dafür Metadaten der SUB Göttingen nutzen, sicherzustellen. ${ }^{32}$

31 S. Kemp, Jennifer und Howard Ratner: Best Practices and Supporting Use Cases for Metadata 2020 Principles. 17.3.2020. http://www.metadata2020.org/blog/2020-03-17-metadata -practices/ (16.10.2020).

32 Niedersächsische Staats- und Universitätsbibliothek Göttingen: Digital Policies: Grundsätze für die digitalen Angebote der SUB Göttingen: Metadaten für Publikationen, Objekte und For- 
Den Qualitätsbegriff greift auch die Library Metadata Policy des Trinity College Dublin auf. ${ }^{33}$ Implizit wird dieser auch in der Policy der Bodlein Libraries erörtert: „This metadata adequately describes the Libraries' collections, wherever these are held, and enables them to be managed well. “34

Ausführlich widmet sich die British Library in der Schrift Foundations for the Future für den Zeitraum zwischen 2019 und 2023 im Sinne einer British Library's Collection Metadata Strategy der Vision „that by 2023 the Library's collection metadata assets will be unified on a single, sustainable, standardsbased infrastructure offering improved options for access, collaboration and open reuse“. ${ }^{35}$ Als eine der Herausforderungen wird die Wahrung des Qualitätsanspruchs bei gleichzeitiger Zunahme des zu betreuenden Umfangs, insbesondere an digitalem Material, angeführt. ${ }^{36}$ Selbstverständlich geht die Nutzung eines standardisierten Ansatzes mit einer gewissen Einschränkung einher, da diese nicht gleichermaßen effizient und effektiv wie eine speziell auf das jeweilige Anliegen abgestimmte Lösung ist, doch ist der Mehrwert für nachhaltige Nutzungsszenarien über die spezifische Domäne hinaus perspektivisch nicht zu verkennen. ${ }^{37}$

Infolgedessen können in einer Metadaten-Richtlinie bzw. -Policy folgende ausgewählte Aspekte hinsichtlich der Inhaltserschließung thematisiert werden: Grundsätzlich sollten Metadaten einer Ressource inhaltserschließende Informationen enthalten, welche digital erstellt und gepflegt werden. Die grundlegende Zielsetzung besteht darin, die Auffindbarkeit der Ressource, Nutzendenbedürfnisse prinzipiell aufgreifend, $\mathrm{zu}$ ermöglichen. Generell sollten Metadatenstandards eingehalten bzw. auf diese zurückgegriffen werden, sodass ausschließlich die Verwendung von - möglichst etablierten - kontrollierten

schungsdaten. https://www.sub.uni-goettingen.de/wir-ueber-uns/portrait/digital-poli cies-grundsaetze-fuer-die-digitalen-angebote-der-sub-goettingen/metadaten/ (16.10.2020).

33 S. The Library of Trinity College, Dublin: Library Metadata Policy. 13.6.2016. https://www. tcd.ie/library/assets/pdf/Policies/Trinity\%20Library\%20Dublin\%20Metadata\%20Policy.pdf (16.10.2020).

34 Bodleian Libraries, University of Oxford: Bodleian Libraries digital policies: Metadata. https://www.bodleian.ox.ac.uk/about/policies/metadata (16.10.2020).

35 Danskin, Alan: Foundations for the future: The British Library's Collection Metadata Strategy, 2019-2023. In: Catalogue and Index (2019) 195. S. 24-33, S. 24.

36 S. The British Library: Foundations for the Future. The British Library's Collection Metadata Strategy, 2019-2023. https://www.bl.uk/bibliographic/pdfs/british-library-collection-metadata -strategy-2019-2023.pdf (16.10.2020), S. 4.

37 S. Metadata and Bibliographic Control, S. 48-49. S. exempl. zu „hauseigenen“ Ansätzen Balakrishnan, Uma und Armin Kühn: Ergebnisse der Umfrage zur sachlichen Erschließung in GBV- und SWB-Teilnehmerbibliotheken. 2019. https://opus.k10plus.de/frontdoor/index/index/ docId/464 (23.10.2020). 
Vokabularen und PIDs erfolgt. Dies wäre öffentlich einsehbar zu dokumentieren. Über standardisierte Schnittstellen sind diese Informationen für Nutzende abrufbar, vorbehaltlich der jeweiligen juristischen Vorgaben. Zuletzt könnte in einer Richtlinie die Prüfung bzw. Umsetzung von Transformationen bzw. Mappings von nicht etablierten zu etablierten Vokabularen vorgeben werden, ggf. auch durch den Einsatz von wohl dokumentierten und ausgewiesenen, automatisierten Verfahren. Wirtschaftliche Aspekte sowie der notwendige Ressourcenbedarf sind hierbei stets einzubeziehen..$^{38}$

Zurückkommend auf die oben erwähnten vier Prinzipien compatible, complete, credible, curated der Initiative Metadata 2020 und die damit verbundenen Handlungsempfehlungen können diese auch Anregungen für zu definierende Qualitätsansprüche in der Erschließungspraxis liefern. ${ }^{39}$ Die Zielsetzung compatible wird durch die Anwendung bereits etablierter Erschließungssysteme bzw. -richtlinien erhöht, um somit kontrollierte Vokabulare in Verbindung mit PID zu etablieren bzw. anzuwenden. Das Ziel complete, wohlwissend um die Ambivalenz hinsichtlich Vollständigkeit, lässt sich durch die Etablierung von Feedback-Mechanismen für Nutzende, auch unter dem Aspekt der Pflege und Entwicklung von kontrollierten Vokabularen, anstreben.

Die den Daten durch die bereitstellende Institution Bibliothek zugeschriebene attributionale Eigenschaft credible könnte durch die öffentlich zugängliche Dokumentation der Verfahren zur Erstellung der inhaltserschließenden Informationen bzw. zur automatisierten Erzeugung für eine nachvollziehbare Herkunft nachgekommen werden. Unter diesem Aspekt ist der Einsatz bzw. die Generierung von inhaltserschließenden Informationen durch Künstliche Intelligenz (KI) und deren Anwendungsnachweis, welcher bisher oftmals nicht vorhanden oder unvollständig ist, zu hinterfragen. Zudem sind entsprechende KIErgebnisse respektive deren Zustandekommen oftmals nur bedingt eingeschränkt nachzuvollziehen bzw. dokumentiert. Um die Glaubwürdigkeit wie auch das Vertrauen in die Institution zu bestätigen, wäre eine kritische Auseinandersetzung mit dem Ziel eines transparenten Umgangs von maschinell erstellten Daten - auch mittels KI erstellter -, unmittelbar in Verbindung mit

38 S. exemplarisch Digital Policies: Grundsätze für die digitalen Angebote der SUB Göttingen. The Library of Trinity College, Dublin: Library Metadata Policy. Bodleian Libraries digital policies: Metadata. S. u. a. zu Wirtschaftlichkeitsaspekten Stumpf, Gerhard: Sacherschließung und Kataloganreicherung. In: Praxishandbuch Bibliotheksmanagement. Hrsg. von Rolf Griebel, Hildegard Schäffler, Konstanze Söllner. Berlin: De Gruyter Saur 2014. S. 357-379, https://doi.org/ 10.1515/9783110303261, S. 375.

39 S. Methods \& Proposal for Metadata Guiding Principles, S. 5. 
einem detaillierten Nachweis - auch für Nutzende einseh- und nachvollziehbar, an den jeweiligen Erschließungsdaten - weiter zu diskutieren. ${ }^{40}$

Den Punkt curated gilt es, mittels regelmäßiger Datenprüfungen und erforderlicher Modifikationen hinsichtlich irriger, zu ergänzender oder zu aktualisierender Angaben dokumentiert umzusetzen. Dies ist sowohl für Erschließungsinformationen als auch für kontrollierte Vokabulare auszuloten.

Verstärkt rücken Ansätze zur maschinellen Erschließung und deren Erprobung ins Zentrum, wobei „klar definierte Qualitätskriterien in der Erschließung“41 gewünscht werden. Jedoch bleiben in explorativen Projekten entsprechende Vorgaben bzw. Annahmen oft vergleichsweise unscharf. ${ }^{42}$ Das Augenmerk wird zunehmend speziell auf die Potentiale und Grenzen für den Einsatz von KI bzw. Deep Learning für die Erschließung gelenkt. ${ }^{43}$ Ausführlich widmete sich auch die Library of Congress dieser Entwicklung, verbunden mit der noch zu vertiefenden Frage: ,What is the role of data in AI/ML [Artificial Intelligence/Machine Learning], and how can we procure, structure, document, and interpret data ethically for AI/ML use cases?"44 Etwaige Antworten wären in einer Metadaten-Richtlinie zukünftig zu berücksichtigen.

40 S. Initiativgruppe des Standardisierungsausschusses: Stellungnahme zur Entwicklung der Inhaltserschließung im D-A-CH-Raum. 8.6.2018. https://www.vdb-online.org/wordpress/ wp-content/uploads/2018/06/Stellungnahme_IE_D-A-CH-Raum.pdf (16.10.2020). Ferner s. in diesem Zusammenhang den diskutablen Ansatz Förster, Frank: Zuweisung von Katalogdatensätzen an Personennormdatensätze mittels Wahrscheinlichkeiten. In: b.i.t.online (2020) 23,2. S. 138-148, https://www.b-i-t-online.de/heft/2020-02-fachbeitrag-foerster.pdf (16.10.2020).

41 Franke-Maier, Michael u. a.: Ein Feuerwerk an Algorithmen und der Startschuss zur Bildung eines Kompetenznetzwerks für maschinelle Erschließung. In: o-bib. Das offene Bibliotheksjournal (2020) 7,1. S. 1-12, https://doi.org/10.5282/o-bib/5565, S. 9.

42 S. exemplarisch Kleppe, Martijn u. a.: Exploration possibilities Automated Generation of Metadata. 2019. http://doi.org/10.5281/zenodo.3375192, S. 14. Für einen Ansatz s. A Framework for Evaluating Automatic Indexing, S. 13-14.

43 S. exemplarisch Ein Feuerwerk an Algorithmen, S. 11-12.

44 Jakeway, Eileen u. a.: LC Labs, Digital Strategy Directorate: Machine Learning + Libraries Summit Event Summary. 13.2.2020. https://labs.loc.gov/static/labs/meta/ML-Event-SummaryFinal-2020-02-13.pdf (16.10.2020), S. 3. Ferner Padilla, Thomas: Responsible Operations: Data Science, Machine Learning, and AI in Libraries. Dublin, OH: OCLC Research 2019, https://doi. org/10.25333/xk7z-9g97. Cordell, Ryan: Machine Learning + Libraries: A Report on the State of the Field; Commissioned by Library of Congress Labs. 14.7.2020. https://labs.loc.gov/static/ labs/work/reports/Cordell-LOC-ML-report.pdf (16.10.2020). 


\section{Umsetzung}

Zwar können mittels Metadaten-Richtlinien entsprechende Vorgaben - u. a. zur Umsetzung eines Strategieprozesses - an einer Institution auch grundlegend zu inhaltserschließenden Informationen formuliert werden, doch letztendlich leitet erst deren Umsetzung, verbunden mit einer Art von Metadata Quality Control Workflow, zu den definierten Zielen. ${ }^{45}$ Als Grundlage für die Realisierung und stetige Verbesserung innerhalb der relevanten Abteilungen könnte hierfür der Rückgriff auf einen kontinuierlichen Kreislauf nach Chou aus dem Jahr 2019, ausgehend von einer strategischen Planung, über Werte und Zielsetzungen, gefolgt von priorisierten und strategisch eingeordneten Handlungs- bzw. Umsetzungsfeldern unterstützend sein. Im Anschluss daran erfolgt im Fortgang des Kreislaufs eine Analyse der Methoden und Ergebnisse. Durch eine kontinuierliche Einschätzung dieser wird letztendlich eine Grundlage für eine mögliche Veränderung bzw. Schärfung der strategischen Planung geschaffen. Somit vollendet dieser Aspekt den Kreislauf. ${ }^{46}$ Ausgehend von diesem letzten Schritt des Kreislaufs könnten daneben Ergebnisse, nach entsprechender Einschätzung, zu einer Modifikation der vorhandenen, übergeordneten Metadaten-Richtlinie führen.

Insbesondere identifizierte Chou als eine der Herausforderungen die Etablierung von Mechanismen zur Umsetzung der strategischen Planungen in das Tagesgeschäft, wie in einer Abteilung für Katalogisierung und Metadaten, und regt u. a. an, dass ,a cataloger's performance can be measured in the context of providing user-centered services, supporting/grasping user experiences, and optimizing the discovery of diverse resources. All of these issues should be communicated by the cataloging managers, cataloging staff, and administrators to keep everyone on the same page. “47

Zudem empfiehlt die Autorin, dass Katalogisierungs- und Metadatentätigkeiten nicht ausschließlich und solitär produzierend aufgefasst werden sollten, da diese weitreichenden Einfluss auf die Zugänglichkeit bzw. Entdeckung der vielfältigen Ressourcen, insbesondere im Zeitalter der globalen, digitalen

45 S. als Ansatz UCLA Library: Library Special Collection: Metadata Quality Control Workflow. https://www.library.ucla.edu/sites/default/files/Guidelines_MetadataQualityControl.pdf (16.10.2020). Nothern Illionois University: Digital Library: Metadata Quality Control Workflow. 15.11.2016. https://digital.lib.niu.edu/policy/metadata/quality-control (16.10.2020). Survey of Benchmarks in Metadata Quality, S. 18-23.

46 S. Chou, Charlene: Purpose-Driven Assessment of Cataloging and Metadata Services: Transforming Broken Links into Linked Data. In: Cataloging \& Classification Quarterly (2019) 57,2-3. S. 135-165, https://doi.org/10.1080/01639374.2019.1571553, S. 158, Figure 8.

47 Purpose-Driven Assessment of Cataloging and Metadata Services, S. 159. 
Wissenschaft, haben. Ferner sollten kontinuierliche Evaluationen in diesem bibliothekarischen Bereich an bestimmte, zu modifizierende Zielsetzungen gebunden sein, erforderliche Kompetenzen von Mitarbeitenden anhand von Analysen identifiziert und ggf. Fortbildungsprogramme entsprechend ausgerichtet sowie durchgeführt werden. Daneben betont Chou in diesem Zusammenhang Transparenz und Kommunikation als Schlüsselfaktoren für das erfolgreiche, bibliothekarische Zusammenarbeiten. ${ }^{48}$

Konkret könnten Qualitätsansprüche anhand eines Metadata Quality Control Workflow, wie im folgenden Beispiel zweistufig, erfolgen. Zuerst könnten entsprechende Schritte während der Eingabe bzw. Bearbeitung nach etwaiger Datenübernahme und unmittelbar im Anschluss eine Evaluation der Daten entsprechend der jeweiligen Vorgaben und potenziell auch (halb-)automatisiert - durchgeführt werden. Diese kann anhand unterschiedlicher Kriterien stattfinden, und somit u. a. die Anzahl der zu überprüfenden Datensätze variieren. ${ }^{49}$ Innerhalb eines Workflows ist daneben die Möglichkeit eines Feedbacks durch Mitarbeitende in Erwägung zu ziehen. Zudem wäre eine Feedback-Kultur für etwaige Ergänzungen und Rückmeldungen von Nutzenden $\mathrm{zu}$ inhaltserschließenden Informationen an Bibliotheksmitarbeitende, verbunden mit einem gesonderten Workflow, denkbar. ${ }^{50}$ Rückmeldungen mit grundsätzlichen Anliegen im Sinne eines nutzendenzentrierten fit for purpose-Ansatzes für inhaltserschließende Informationen sollten - nach hinreichender Prüfung - letztendlich ebenfalls in eine, nach Bedarf notwendige, Modifikation der bibliotheksspezifischen Metadaten-Richtlinie einfließen.

Inwiefern die seit beinahe zwanzig Jahren verfolgten Entwicklungen rund um das Semantic Web, Linked Data bzw. LOUD, ${ }^{51} \mathrm{im}$ bibliothekarischen Umfeld

48 S. Purpose-Driven Assessment of Cataloging and Metadata Services, S. 157-161.

49 S. als Ansatz UCLA Library: Metadata Quality Control Workflow. Digital Library: Metadata Quality Control Workflow.

50 S. Best Practices and Supporting Use Cases for Metadata 2020 Principles. Purpose-Driven Assessment of Cataloging and Metadata Services, S. 138-139, 159, 161.

51 S. Berners-Lee, Tim u. a.: The Semantic Web: a new form of Web content that is meaningful to computers will unleash a revolution of new possibilities. In: Scientific American (2001) 284,5. S. 34-43. Kritisch s. Marshall, Catherine C. und Frank M. Shipman: Which semantic web? In: HYPERTEXT '03: the Fourteenth ACM Conference on Hypertext and Hypermedia, August 26-30, 2003, Nottingham, UK. New York, NY: Assoc. for Computing Machinery 2003. S. 57-66, S. 62-63, 65. Target, Sinclair: Whatever Happened to the Semantic Web? 27.5.2018. https://twobithistory.org/2018/05/27/semantic-web.html (16.10.2020). Sanderson, Rob: Shout it Out: LOUD. 2018. https://www.slideshare.net/Europeana/shout-it-out-loud-by-rob-san derson-europeanatech-conference-2018-98225909 (16.10.2020). Welche nachhaltigen Entwicklungen sich durch das von Tim Berners-Lee geleitete „Project Solid“, https://solid.mit.edu/ (16.10.2020), ergeben, bleibt zu beobachten. Für eine Rezeption aus den Digital Humanities 
u. a. durch BIBFRAME 2.0 vertreten, einen Mehrwert, ebenfalls für die Datenqualität von inhaltserschließenden Angaben, darstellen werden, befindet sich weiterhin in einer intensiven Diskussion und einer letztlich damit verbundenen Abwägung einer breiten Anwendung. ${ }^{52}$ Ob die Herausforderungen um die teilweise eingeleiteten Überführungen von Klassifikationssystemen oder Normdatendiensten nachhaltig gelöst und diese Dienste dauerhaft angeboten werden, bleibt folglich zu beobachten, zumal das aus der Informatik stammende GIGOPrinzip (Garbage In, Garbage Out) auch für diese Ansätze nicht an Bedeutung verliert. ${ }^{53}$ Nicht nur sind die durch die jeweilige Institution nachhaltig aufzubringenden Ressourcen im Zusammenhang mit Linked-(Open)-Data-Angeboten (L(O)D) pragmatisch abzuschätzen, sondern ist im Vorwege die Zielsetzung einer Bibliothek aufgrund elaborierter Nutzendenbedürfnisse hinsichtlich L(O)D stets zu konkretisieren und erst nach erfolgter Abschätzung der Aspekt L(O)D in einer Metadaten-Richtlinie zu verankern.

s. Dienhart, Michaela und Nils Geißler: Veranstaltungspost: LOUD: Turn up your data! 28.9.2020. https://dhc.hypotheses.org/1397 (16.10.2020).

52 S. Container - Content - Context, S. 127-128; Altenhöner, Reinhard: Jenseits der Cloud: Metadaten- und Datenmanagement in der bibliothekarischen Infrastruktur: Teil 1. In: Bibliotheksdienst (2015) 49,7. S. 677-695, https://doi.org/10.1515/bd-2015-0080, S. 683-684. Ferner Tennant, Roy: MARC Must Die. In: Library Journal [Print 2002]. 21.5.2010. https://www.library journal.com/?detailStory=marc-must-die (16.10.2020). Kroeger, Angela: The Road to BIBFRAME: The Evolution of the Idea of Bibliographic Transition into a Post-MARC Future. In: Cataloging \& Classification Quarterly (2013) 51,8. S. 873-890, https://doi.org/10.1080/ 01639374.2013.823584, S. 884-885. Wallis, Richard: Getting [library data] from there to here... In: Catalogue and Index (2020) 199. S. 3-8. https://cdn.ymaws.com/www.cilip.org.uk/ resource/collection/5F814B6D-500C-42B2-9D5F-E6E3C550C24A/C\&I199Wallis_There_to_here. pdf (16.10.2020). Für das Archivwesen s. Gniffke, David: Semantic Web und Records in Contexts (RiC). In: Archivwelt-Archivwissenschaftliches Blog der Archivschule Marburg. 16.3.2020. https://archivwelt.hypotheses.org/1982 (16.10.2020).

53 S. Hanke, Mirko: Bibliothekarische Klassifikationssysteme im semantischen Web: Zu Chancen und Problemen von Linked-Data-Repräsentationen ausgewählter Klassifikationssysteme. In: Perspektive Bibliothek (2014) 3,2. S. 91-119, https://doi.org/10.11588/PB.2014.2.16808. Kasprzik, Anna und Jürgen Kett: Vorschläge für eine Weiterentwicklung der Sacherschließung und Schritte zur fortgesetzten strukturellen Aufwertung der GND. In: o-bib. Das offene Bibliotheksjournal (2018) 5,4. S. 127-140, https://doi.org/10.5282/o-bib/2018H4S127-140. Zuletzt Frosterus, Matias u. a.: Linked Open Data: Impressions \& Challenges Among Europe’s Research Libraries. 2020. http://doi.org/10.5281/zenodo.3647844. Smith-Yoshimura, Karen: Transitioning to the Next Generation of Metadata. Dublin, OH: OCLC Research 2020, https://doi.org/10.25333/ rqgd-b343, S. 14-15. 


\section{Fazit}

Inhaltserschließende Aufgaben sollten nicht ausschließlich Nutzenden eines bibliothekarischen Service übertragen werden, da entsprechende Kompetenzen, wie zu Beginn dieses Beitrags aufgezeigt, oftmals nicht vorhanden sind, und auch in den seltensten Fällen Teil des originären Forschungsvorhabens bzw. -interesses sind bzw. werden. Die Betreuung der, durchaus auch (halb-)automatisiert erzeugten, inhaltserschließenden Metadaten sollte fachlich kompetenten Mitarbeitenden einer Bibliothek - welche neben der aktuellen Entwicklung weiterhin ,eine Einrichtung [ist], die unter archivarischen, ökonomischen und synoptischen Gesichtspunkten publizierte Information für die Benutzer sammelt, ordnet und verfügbar macht“54 - obliegen, um letztendlich zeitgemäße Datendienstleistungen nutzendenzentriert und auf bibliothekarisch angemessenem Qualitätsniveau anbieten zu können. ${ }^{55}$

Die Formulierung, Veröffentlichung - auch im Sinne der Transparenz und praktische Umsetzung einer bibliotheksspezifischen Metadaten-Richtlinie bzw. -Policy unter Einbeziehung der vier Prinzipien compatible, complete, credible, curated kann als eine Grundlage für nutzendenzentrierte Metadatenangebote - einschließlich inhaltserschließender Informationen mit entsprechenden Qualitätsmerkmalen - dienen.

\section{Literaturverzeichnis}

Allianz der deutschen Wissenschaftsorganisationen: „Wege zur digitalen Qualifikation“: ein Diskussionspapier. 2020. https://doi.org/10.2312/allianzoa.038.

Altenhöner, Reinhard: Jenseits der Cloud: Metadaten- und Datenmanagement in der bibliothekarischen Infrastruktur: Teil 1. In: Bibliotheksdienst (2015) Bd. 49 H. 7. S. 677-695. https://doi.org/10.1515/bd-2015-0080.

Bach, F. u. a.: Muster-Richtlinie Nachhaltige Forschungssoftware an den Helmholtz-Zentren. Potsdam 2019. https://doi.org/10.2312/OS.HELMHOLTZ.007.

54 Ewert, Gisela und Walther Umstätter: Bibliotheken. Die Definition der Bibliothek. In: Bibliotheksdienst (1999) 33,6. S. 957-971, https://doi.org/10.1515/bd.1999.33.6.957, S. 966. Ferner Bonte, Achim: Was ist eine Bibliothek? Physische Bibliotheken im digitalen Zeitalter. In: ABI Technik (2015) 35,2. S. 95-104, https://doi.org/10.1515/abitech-2015-0019.

55 S. Beckmann, Regine und Vivien Petras: Inhaltserschließung am Institut für Bibliotheksund Informationswissenschaft der Humboldt-Universität zu Berlin: Inhaltserschließung in der Ausbildung bleibt wichtig, Schwerpunkte ändern sich. In: BuB: Forum Bibliothek und Information (2018) 70,1. S. 41-43, https://nbn-resolving.org/urn:nbn:de:0290-opus4-160798, S. 43. 
Bade, David: The Perfect Bibliographic Record: Platonic Ideal, Rhetorical Strategy or Nonsense? In: Cataloging \& Classification Quarterly (2008) Bd. 46 H. 1. S. 109-133. https://doi.org/10.1080/01639370802183081.

Balakrishnan, Uma und Armin Kühn: Ergebnisse der Umfrage zur sachlichen Erschließung in GBV- und SWB-Teilnehmerbibliotheken. 2019. https://opus.k10plus.de/frontdoor/index/ index/docld/464 (23.10.2020).

Balatsoukas, Panos u. a.: A Method for Examining Metadata Quality in Open Research Datasets Using the OAI-PMH and SQL Queries: the Case of the Dublin Core 'Subject' Element and Suggestions for User-Centred Metadata Annotation Design. In: International Journal of Metadata, Semantics and Ontologies (2018) Bd. 13 H. 1. S. 1-8. https://doi. org/10.1504/IJMSO.2018.096444.

Batini, Carlo und Monica Scannapieca: Data quality: concepts, methodologies and techniques. Berlin, New York: Springer 2006 (Data-centric systems and applications).

Beckmann, Regine und Vivien Petras: Inhaltserschließung am Institut für Bibliotheks- und Informationswissenschaft der Humboldt-Universität zu Berlin: Inhaltserschließung in der Ausbildung bleibt wichtig, Schwerpunkte ändern sich. In: BuB: Forum Bibliothek und Information (2018) Jg. 70 H. 1. S. 41-43. https://nbn-resolving.org/urn:nbn: de:0290-opus4-160798.

Berners-Lee, Tim u. a.: The Semantic Web: a new form of Web content that is meaningful to computers will unleash a revolution of new possibilities. In: Scientific American (2001) Bd. 284 H. 5. S. 34-43.

Bodleian Libraries, University of Oxford: Bodleian Libraries digital policies: Metadata. https:// www.bodleian.ox.ac.uk/about/policies/metadata (16.10.2020).

Bonte, Achim: Was ist eine Bibliothek? Physische Bibliotheken im digitalen Zeitalter. In: ABI Technik (2015) Bd. 35 H. 2. S. 95-104. https://doi.org/10.1515/abitech-2015-0019.

Broeder, Daan u. a.: SSHOC D3.1 Report on SSHOC (meta)data interoperability problems. 10.12.2019. https://doi.org/10.5281/zenodo.3569868.

Chou, Charlene: Purpose-Driven Assessment of Cataloging and Metadata Services: Transforming Broken Links into Linked Data. In: Cataloging \& Classification Quarterly (2019) Bd. 57. H. 2-3. S. 135-165. https://doi.org/10.1080/01639374.2019.1571553.

Cordell, Ryan: Machine Learning + Libraries: A Report on the State of the Field; Commissioned by Library of Congress Labs. 14.7.2020. https://labs.loc.gov/static/labs/work/reports/ Cordell-LOC-ML-report.pdf (16.10.2020).

Danskin, Alan: Foundations for the future: The British Library's Collection Metadata Strategy, 2019-2023. In: Catalogue and Index (2019) H. 195. S. 24-33.

DFG: Leitlinien zur Sicherung guter wissenschaftlicher Praxis - Kodex. Bonn 2019. https:// www.dfg.de/download/pdf/foerderung/rechtliche_rahmenbedingungen/gute_ wissenschaftliche_praxis/kodex_gwp.pdf (16.10.2020).

Dienhart, Michaela und Nils Geißler: Veranstaltungspost: LOUD: Turn up your data! 28.9.2020. https://dhc.hypotheses.org/1397 (16.10.2020).

Doctorow, Cory: Metacrap: Putting the torch to seven straw-men of the meta-utopia. 26.8.2001. https://people.well.com/user/doctorow/metacrap.htm (16.10.2020).

Döhl, Frédéric: Digital Humanities und Bibliotheken. Über technisch-organisatorische Infrastruktur hinausgedacht. In: Zeitschrift für Bibliothekswesen und Bibliographie (2019) Jg. 66 H. 1. S. 4-18, https://doi.org/10.3196/186429501966114.

Ede, Stuart: Fitness for purpose: the future evolution of bibliographic records and their delivery. In: Catalogue and Index (1995) H. 116. S. 1-3. 
Einwächter, Sophie G. u. a.: Lessons learned - Thesen zur FDM-Kompetenzausbildung: Erkenntnisse aus dem interdisziplinären Vernetzungstreffen der vom BMBF geförderten Projekte eeFDM Jena, FDMentor, FOKUS, PODMAN und UniLLAB am 30. und 31. Januar 2019 in Marburg. In: Bausteine Forschungsdatenmanagement (2020) Nr. 1. S. 8-15. https://doi. org/10.17192/bfdm.2020.1.8101.

Ewert, Gisela und Walther Umstätter: Bibliotheken. Die Definition der Bibliothek. In: Bibliotheksdienst (1999) Bd. 33 H. 6. S. 957-971. https://doi.org/10.1515/bd.1999.33.6.957.

Franke-Maier, Michael: Anforderungen an die Qualität der Inhaltserschließung im Spannungsfeld von intellektuell und automatisch erzeugten Metadaten. In: ABI-Technik (2018) Bd. 38 H. 4. S. 327-331. https://doi.org/10.1515/abitech-2018-4005.

Franke-Maier, Michael u. a.: Ein Feuerwerk an Algorithmen und der Startschuss zur Bildung eines Kompetenznetzwerks für maschinelle Erschließung. In: o-bib. Das offene Bibliotheksjournal (2020) Bd. 7 H. 1. S. 1-12. https://doi.org/10.5282/o-bib/5565.

Frosterus, Matias u. a.: Linked Open Data: Impressions \& Challenges Among Europe's Research Libraries. 2020. http://doi.org/10.5281/zenodo.3647844.

Förster, Frank: Zuweisung von Katalogdatensätzen an Personennormdatensätze mittels Wahrscheinlichkeiten. In: b.i.t.online (2020) Jg. 23 Nr. 2. S. 138-148. https://www.b-i-t-online. de/heft/2020-02-fachbeitrag-foerster.pdf (16.10.2020).

Gentry, Steven u. a.: Survey of Benchmarks in Metadata Quality: Initial Findings. 2020. https://digital.library.unt.edu/ark:/67531/metadc1637685/ (16.10.2020).

Gniffke, David: Semantic Web und Records in Contexts (RiC). In: Archivwelt-Archivwissenschaftliches Blog der Archivschule Marburg. 16.3.2020. https://archivwelt.hypotheses. org/1982 (16.10.2020).

Golub, Koraljka u. a.: A Framework for Evaluating Automatic Indexing or Classification in the Context of Retrieval. In: Journal of the Association for Information Science and Technology (2016) Bd. 67 H. 1. S. 3-29. https://doi.org/10.1002/asi.23600.

Gradmann, Stefan: Container - Content - Context: zur Evolution bibliothekarischer Metadaten von Katalogdaten zu Library Linked Data. In: Handbuch Bibliothek: Geschichte, Aufgaben, Perspektiven. Hrsg. von Konrad Umlauf und Stefan Gradmann. Stuttgart, Weimar: Metzler 2012. S. 121-128.

Hanke, Mirko: Bibliothekarische Klassifikationssysteme im semantischen Web: Zu Chancen und Problemen von Linked-Data-Repräsentationen ausgewählter Klassifikationssysteme. In: Perspektive Bibliothek (2014) Bd. 3 Nr. 2. S. 91-119. https://doi.org/10.11588/PB. 2014.2.16808.

Howarth, Lynne C.: Metadata and Bibliographic Control: Soul-Mates or Two Solitudes? In: Cataloging \& Classification Quarterly (2005) Bd. 40 H. 3-4. S. 37-56. https://doi.org/10. 1300/J104v40n03_03.

Initiativgruppe des Standardisierungsausschusses: Stellungnahme zur Entwicklung der Inhaltserschließung im D-A-CH-Raum. 8.6.2018. https://www.vdb-online.org/wordpress/ wp-content/uploads/2018/06/Stellungnahme_IE_D-A-CH-Raum.pdf (16.10.2020).

Jakeway, Eileen u. a.: LC Labs, Digital Strategy Directorate: Machine Learning + Libraries Summit Event Summary. 13.2.2020. https://labs.loc.gov/static/labs/meta/ML-Event-Sum mary-Final-2020-02-13.pdf (16.10.2020).

Kaiser, Kathryn u. a.: Methods \& Proposal for Metadata Guiding Principles for Scholarly Communications. In: Research Ideas and Outcomes (2020) Nr. 6. e53916. https://doi.org/10. 3897/rio.6.e53916. 
Kasprzik, Anna und Jürgen Kett: Vorschläge für eine Weiterentwicklung der Sacherschließung und Schritte zur fortgesetzten strukturellen Aufwertung der GND. In: o-bib. Das offene Bibliotheksjournal (2018) Bd. 5 H. 4. S. 127-140. https://doi.org/10.5282/o-bib/ 2018H4S127-140.

Kemp, Jennifer und Howard Ratner: Best Practices and Supporting Use Cases for Metadata 2020 Principles. 17.3.2020. http://www.metadata2020.org/blog/2020-03-17-metadatapractices/ (16.10.2020).

Kleppe, Martijn u. a.: Exploration possibilities Automated Generation of Metadata. 2019. http://doi.org/10.5281/zenodo.3375192.

Klimpel, Paul: Kulturelles Erbe digital: eine kleine Rechtsfibel. Berlin: digiS Forschungs- und Kompetenzzentrum Digitalisierung 2020. https://doi.org/10.12752/2.0.004.0.

Kroeger, Angela: The Road to BIBFRAME: The Evolution of the Idea of Bibliographic Transition into a Post-MARC Future. In: Cataloging \& Classification Quarterly (2013) Bd. 51 H. 8. S. 873-890. https://doi.org/10.1080/01639374.2013.823584.

Lin, Dawei u. a.: The TRUST Principles for Digital Repositories. In: Scientific Data (2020) Bd. 7 Artikelnr. 144. https://doi.org/10.1038/s41597-020-0486-7.

Mache, Beata: Meine erste Kollektion im DARIAH-DE Repository. DHdBlog. 7.12.2017. https:// dhd-blog.org/?p=8798 (16.10.2020).

Marshall, Catherine C. und Frank M. Shipman: Which semantic web? In: HYPERTEXT '03: the Fourteenth ACM Conference on Hypertext and Hypermedia, August 26-30, 2003. Nottingham, UK. New York, NY: Assoc. for Computing Machinery 2003. S. 57-66.

Müller, Gerhard: Die Normdatei als ein Mittel in der Erschließung von Archivbeständen. In: Brandenburgische Archive (2015) H. 32. S. 8-16.

Niedersächsische Staats- und Universitätsbibliothek Göttingen: Digital Policies: Grundsätze für die digitalen Angebote der SUB Göttingen: Metadaten für Publikationen, Objekte und Forschungsdaten. https://www.sub.uni-goettingen.de/wir-ueber-uns/portrait/digital-poli cies-grundsaetze-fuer-die-digitalen-angebote-der-sub-goettingen/metadaten/ (16.10.2020).

Nothern Illionois University: Digital Library: Metadata Quality Control Workflow. 15.11.2016. https://digital.lib.niu.edu/policy/metadata/quality-control (16.10.2020).

Ochoa, Xavier und Erik Duval: Automatic Evaluation of Metadata Quality in Digital Repositories. In: International Journal on Digital Libraries (2009) Bd. 10 S. 67-91. https://doi.org/10. 1007/s00799-009-0054-4.

Padilla, Thomas: Responsible Operations: Data Science, Machine Learning, and Al in Libraries. Dublin, OH: OCLC Research 2019. https://doi.org/10.25333/xk7z-9g97.

Paulmann, Johannes und Eva Schlotheuber: Digitale Wissensordnung und Datenqualität: Herausforderungen, Anforderungen und Beitrag historisch arbeitender Wissenschaften. In: Archivar (2020) Jg. 73 H. 1. S. 9-12.

Peer, Limor u. a.: Committing to Data Quality Review. In: International Journal of Digital Curation (2014) Bd. 9 H. 1. S. 263-291. https://doi.org/10.2218/ijdc.v9i1.317.

Project Solid. https://solid.mit.edu/ (16.10.2020).

Rat für Informationsinfrastrukturen (Hrsg.): Herausforderung Datenqualität. Empfehlungen zur Zukunftsfähigkeit von Forschung im digitalen Wandel. 2. Aufl. Göttingen 2019. http:// www.rfii.de/?p=4043 (16.10.2020).

Report and Recommendations from the Europeana Task Force on Metadata Quality. Den Haag 2015. http://pro.europeana.eu/files/Europeana_Professional/Publications/Metadata\% 20Quality\%20Report.pdf (16.10.2020). 
Riley, Jenn: Seeing Standards: A Visualization of the Metadata Universe. 2010. http://jennriley.com/metadatamap/ (16.10.2020).

Riley, Jenn: Understanding Metadata: What Is Metadata, and What Is It For? Baltimore 2017. http://www.niso.org/publications/understanding-metadata-riley (16.10.2020).

Riva, Pat u. a.: IFLA Library Reference Model (LRM). Den Haag 2017. https://www.ifla.org/files/ assets/cataloguing/frbr-Irm/ifla-Irm-august-2017_rev201712.pdf (16.10.2020).

Sanderson, Rob: Shout it Out: LOUD. 2018. https://www.slideshare.net/Europeana/shout-i t-out-loud-by-rob-sanderson-europeanatech-conference-2018-98225909 (16.10.2020).

Schulz, Julian u. a.: Standardisierung eines Standards: Warum und wie ein Best-Practice-Guide für das Metadatenschema DataCite entstand. In: Korpus im Text (2020). http://www.kit. gwi.uni-muenchen.de/? $p=42800 \& v=1$ (16.10.2020).

Shankar, G. und Stephanie Watts: A relevant, believable approach for data quality assessment. In: Proceedings of the Eighth International Conference on Information Quality. 2003. S. 178-189, http://mitiq.mit.edu/ICIQ/Documents/IQ\%20Conference\%202003/Pa pers/ARelevantBelievableApproachForDQAssessment.pdf (16.10.2020).

Shankaranarayanan, G. und Roger Blake: From Content to Context: The Evolution and Growth of Data Quality Research. In: Journal of Data and Information Quality (2017) Bd. 8 Nr. 2. S. 1-28. https://doi.org/10.1145/2996198.

Smith-Yoshimura, Karen: Transitioning to the Next Generation of Metadata. Dublin, OH: OCLC Research 2020. https://doi.org/10.25333/rqgd-b343.

Stocker, Markus u. a.: Persistent Identification Of Instruments. In: arXiv:2003.12958 [cs.DL], 29.3.2020. http://arxiv.org/abs/2003.12958.

Stumpf, Gerhard: Sacherschließung und Kataloganreicherung. In: Praxishandbuch Bibliotheksmanagement. Hrsg. von Rolf Griebel, Hildegard Schäffler, Konstanze Söllner. Berlin: De Gruyter Saur 2014. S. 357-379. https://doi.org/10.1515/9783110303261.

Suominen, Osma u. a.: From MARC Silos to Linked Data Silos? In: o-bib. Das offene Bibliotheksjournal (2017) Bd. 4 H. 2. S. 1-13. https://doi.org/10.5282/O-BIB/2017H2S1-13.

Target, Sinclair: Whatever Happened to the Semantic Web? 27.5.2018. https://twobithistory. org/2018/05/27/semantic-web.html (16.10.2020).

Tennant, Roy: MARC Must Die. In: Library Journal [Print 2002]. 21.5.2010. https://www. libraryjournal.com/?detailStory=marc-must-die (16.10.2020).

The British Library: Foundations for the Future. The British Library's Collection Metadata Strategy, 2019-2023. https://www.bl.uk/bibliographic/pdfs/british-library-collection-me tadata-strategy-2019-2023.pdf (16.10.2020).

The Library of Trinity College, Dublin: Library Metadata Policy. 13.6.2016. https://www.tcd.ie/ library/assets/pdf/Policies/Trinity\%20Library\%20Dublin\%20Metadata\%20Policy.pdf (16.10.2020).

Thomas, Sarah E.: Quality in bibliographic control. In: Library Trends (1996) Bd. 44 Nr. 3. S. 491-505.

UCLA Library: Library Special Collection: Metadata Quality Control Workflow. https://www. library.ucla.edu/sites/default/files/Guidelines_MetadataQualityControl.pdf (16.10.2020).

Wallis, Richard: Getting [library data] from there to here... In: Catalogue and Index (2020) H. 199. S. 3-8. https://cdn.ymaws.com/www.cilip.org.uk/resource/collection/ 5F814B6D-500C-42B2-9D5F-E6E3C550C24A/C\&1199Wallis_There_to_here.pdf (16.10.2020). 
Wang, Richard Y. und Diane M. Strong: Beyond Accuracy: What Data Quality Means to Data Consumers. In: Journal of Management Information Systems (1996) Bd. 12 H. 4. S. 5-33. https://www.jstor.org/stable/40398176.

Wilkinson, Mark D. u. a.: The FAIR Guiding Principles for Scientific Data Management and Stewardship. In: Scientific Data (2016) Bd. 3 Artikelnr. 160018. https://doi.org/10.1038/sda ta.2016.18. 